\title{
Sistema para Classificação de Infestação Parasitária em Pequenos Ruminantes
}

\author{
Lucas F. de Souza, Lucas B. Mior, Márcio H. Costa e Beatriz Riet-Correa
}

Resumo-A pecuária constitui parte significativa do produto interno bruto brasileiro, sendo que o controle sanitário possui importante impacto nos custos do manejo do rebanho. Com o intuito de contribuir para a incorporação de novas tecnologias nesta cadeia econômica, este trabalho apresenta o desenvolvimento de um aplicativo móvel para decisão de tratamento contra infestação parasitária em pequenos ruminantes. Inicialmente, é descrita uma proposta de programa para gerenciamento e aquisição de imagens, baseado no sistema operacional android, e em sequência é apresentado um classificador baseado no método Famacha. Resultados iniciais apresentam uma acurácia de $83 \%$, indicando desempenho superior a propostas anteriores.

Palavras-Chave-Famacha, pequenos ruminantes, parasitas. classificador, aprendizado de máquina.

Abstract- Livestock is a significant part of the Brazilian gross domestic product, and sanitary control has an important impact on herd management costs. In order to contribute to the incorporation of new technologies in this economic chain, this work presents the development of a mobile application for decision about treatment against parasitic infestation in small ruminants. Initially, the proposed software for managing and image acquisition, based on the android system, is described. Following, a neural classifier based on the Famacha method is presented. Initial results show an accuracy of $83 \%$, indicating a performance superior to previous proposals.

Keywords - Famacha, small ruminants, parasites, classifier, machine learning.

\section{INTRODUÇÃO}

A criação de pequenos ruminantes tem apresentado crescimento sustentado ao longo das últimas décadas, mostrando-se como alternativa viável para a geração de emprego e renda tanto na agricultura familiar quanto no agronegócio. Os principais fatores limitantes da produtividade na ovinocultura e na caprinocultura são as doenças causadas por parasitas gastrointestinais (PGI) [1]. Os PGI causam consideráveis perdas econômicas, pois além de reduzirem o potencial produtivo, através da diminuição do ganho de peso e da produção de lã e leite, podem causar a morte de animais jovens e matrizes no periparto [2].

Embora existam diversos gêneros e espécies de PGI que possam infectar pequenos ruminantes, o Haemonchus Contortus é o de maior importância devido à sua prevalência e grau de patogenicidade que, através de sua ação hematófaga, provoca anemia e hipoproteinemia. Cada parasita pode remover até $0,05 \mathrm{ml}$ de sangue por dia. Em infecções graves,

Lucas Souza, Lucas Mior e Márcio Costa, Universidade Federal de Santa Catarina, Departamento de Engenharia Elétrica e Eletrônica, Florianópolis-SC. Beatriz Riet-Correa, Universidade Federal do Rio Grande do Sul, Faculdade de Veterinária, Porto Alegre-RS. E-mails: lucas.fiamoncini00@gmail.com, lucas.mior@tutamail.com, costa@eel.ufsc.br, beatrizriet@hotmail.com. pode haver uma perda diária de $6 \%$ a $25 \%$ dos eritrócitos [3].

Durante décadas, os anti-helmínticos foram utilizados como única estratégia de controle das parasitoses, entretanto, a consequência do uso indiscriminado desses químicos foi o desenvolvimento da resistência anti-helmíntica (RA) [4]. A RA é definida como sendo a capacidade de uma população de parasitas de sobreviver a doses de anti-helmínticos que poderiam ser letais para populações susceptíveis [5]. A RA tem se tornado um problema mundial na criação de pequenos ruminantes, e tem sido relatada em diversos países [6] [7] [8], incluindo no Brasil [9] [10] [11]. Como resultado, a RA pode não apenas encurtar a vida útil dos compostos químicos utilizados, como também inutilizar esta estratégia de manejo [12].

As técnicas de controle parasitário utilizadas até o momento têm levado ao aparecimento da resistência simultânea a vários anti-helmínticos (resistência múltipla), o que resulta em altos custos de tratamento e pode inviabilizar a ovino/caprinocultura em uma região. Para debelar o avanço da resistência, uma das ferramentas mais eficientes é a terapêutica seletiva, na qual é tratada somente uma parte do rebanho. Em geral, apenas $20 \%$ a $30 \%$ dos animais em um rebanho carregam de $70 \%$ a $80 \%$ dos parasitas adultos [13]. Portanto, a identificação desses animais e a administração de anti-helmíntico somente para essa parcela da população aumenta a refugia (população parasita que não foi exposta ao processo de seleção pelas drogas), diminuindo o risco de resistência anti-helmíntica [4].

Neste trabalho, é apresentado o desenvolvimento de um aplicativo para sistemas móveis (aparelhos celulares) para classificação do grau de infestação parasitária em pequenos ruminantes. Inicialmente, é descrita uma proposta de programa para gerenciamento e aquisição de imagens, baseada no sistema operacional android, e, em sequência, é apresentado um classificador de indicação de tratamento de anemia, baseado na análise da imagem da mucosa conjuntiva ocular. Animais de cinco rebanhos distintos foram analisados por um médico veterinário com ampla experiência na realização do método Famacha $^{\odot}$. A acurácia, precisão e sensibilidade do classificador proposto são apresentadas e comparadas com resultados disponíveis na literatura, demonstrando o desempenho do sistema.

A seção II apresenta o método Famacha ${ }^{\circledR}$ para classificação visual do grau de anemia, enquanto que na seção III é descrito o sistema proposto. Na seção IV são apresentados os materiais e métodos utilizados para o desenvolvimento do aplicativo móvel e do classificador. A seção $\mathrm{V}$ apresenta os resultados obtidos e comparações com a literatura. Finalizando, as seções VI e VII apresentam a discussão e a conclusão do trabalho. 


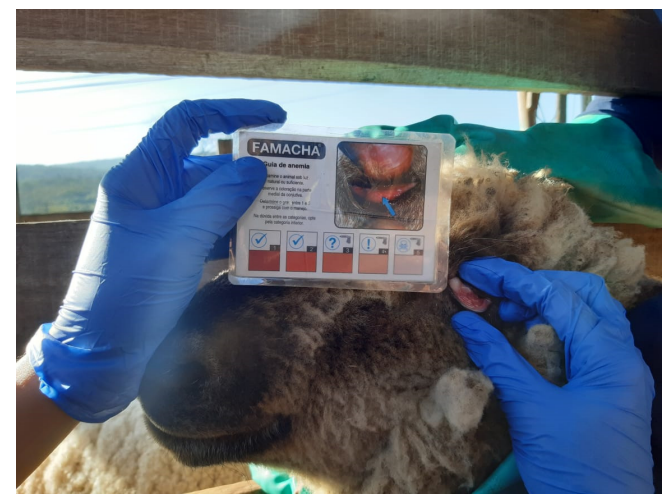

Fig. 1. Utilização do método FAMACHA ${ }^{\odot}$

TABELA I. VALORES DE HEMATÓCRITO, COLORAÇÃO DA MUCOSA OCULAR E RECOMENDAÇÃO DE TRATAMENTO SEGUNDO O MÉTODO FAMACHA ${ }^{\circledR}[16]$.

\begin{tabular}{|c|c|c|c|}
\hline Grau & Hematócrito & Coloração & Tratamento \\
\hline 1 & $>28 \%$ & vermelha & não \\
\hline 2 & 23 a $27 \%$ & rósea-vermelha & não \\
\hline 3 & 18 a $22 \%$ & rósea & $\operatorname{sim}$ \\
\hline 4 & 13 a $17 \%$ & rósea-pálida & $\operatorname{sim}$ \\
\hline 5 & $\leq 12$ & pálida & $\operatorname{sim}$ \\
\hline
\end{tabular}

\section{MÉTODO FAMACHA}

O método Famacha ${ }^{\odot}$ (Faffa Malan Chart) foi desenvolvido na África do Sul pelos pesquisadores François Malan, Gareth Bath e Jan van Wyk, sendo considerado o tratamento mais utilizado no mundo para infestação parasitária em rebanhos de ovinos e caprinos [15]. Seu princípio de funcionamento está baseado na correlação encontrada entre os valores de hematócrito e as diferentes colorações da mucosa conjuntiva ocular $(\rho=0,8$; com confiabilidade superior a 95\%). Assumese, portanto, que ovinos ou caprinos infectados pelo Haemonchus Contortus podem ser identificados pela comparação da coloração da mucosa ocular com uma escala de cores apresentada em um cartão guia ilustrativo (Fig. 1) [16]. Dessa forma, é possível identificar animais que precisam tratamento sem a necessidade de recursos laboratoriais.

A TABELA I apresenta os cinco graus de classificação do método, estando associados a duas classes de tratamento: (a) animais com graus 1 e 2 são considerados saudáveis e, portanto, não recebem medicação; (b) o grau 3 é considerado representativo de grau de anemia limítrofe, sendo a aplicação de anti-helmíntico recomendada, enquanto que nos graus 4 e 5 a medicação é imprescindível.

A eficácia do método Famacha $^{\odot}$ tem sido estudada e validada por vários pesquisadores em diferentes países [17]. A adoção deste método tem resultado na redução do número de doses e do custo com medicação antiparasitária, pois somente os animais que apresentam anemia são tratados [17] [18]. Em estudos realizados no Brasil, foi verificado que este método pode reduzir em até $79,5 \%$ as aplicações com medicação antiparasitária em ovinos [15]. Portanto, apresenta as seguintes vantagens: a diminuição de produtos químicos despejados na natureza; o menor número de animais submetidos a tratamento químico; o menor número de parasitas sofrendo mutações e criando resistência à medicação; bem como a manutenção da eficácia das drogas antiparasitárias comerciais disponíveis [18].
O método Famacha $^{\odot}$ deve ser implantado por médico veterinário treinado, responsável pela difusão da técnica ao produtor rural. Entretanto, o desempenho do método pode ser influenciado por fatores como: a experiência do aplicador; as condições de iluminação; e a idade e sexo do animal. Dessa forma, apresenta uma taxa de falha não desprezível, principalmente em função do erro humano ao realizar a comparação da mucosa com o cartão guia. A partir do exposto, sistemas baseados em técnicas de aprendizado de máquina poderiam propiciar taxas de classificação comparáveis às obtidas por especialistas humanos, como ocorre em aplicações correlatas [19], permitindo a diminuição dos custos envolvidos.

Apesar da importância do método Famacha ${ }^{\circledR}$, uma extensa revisão da bibliografia indica um pequeno número de propostas para sua implementação informatizada [18] [20] [21]. Os resultados apresentados em [18] e [20] apresentam baixos percentuais de acurácia, enquanto que a patente descrita em [21] foi abandonada. Como resultado, existe uma substancial lacuna em relação às oportunidades tecnológicas associadas.

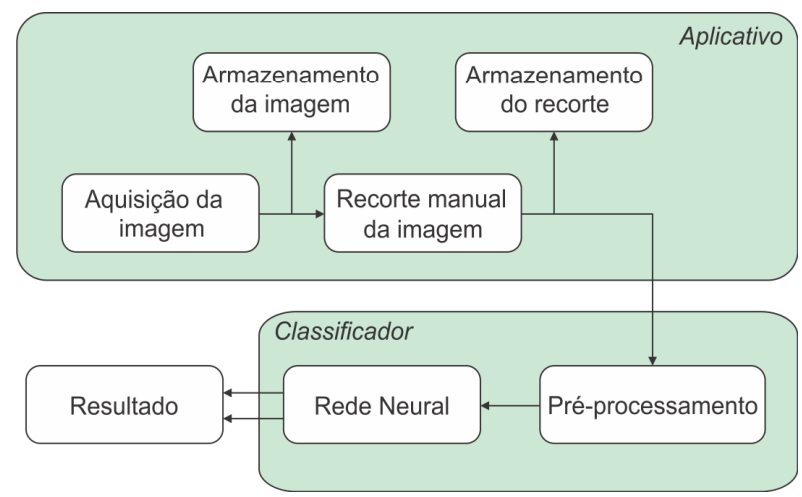

Fig. 2. Diagrama de interconexão dos sistemas de aquisição e classificação.

\section{Sistema Proposto}

O sistema proposto consiste em um aplicativo para dispositivos móveis celulares, baseado no sistema operacional android, e em um classificador da coloração da mucosa conjuntiva ocular, baseado em redes neurais. O diagrama ilustrativo de funcionamento é apresentado na Fig. 2.

\section{A. Aplicativo para Aquisição de Imagens}

$\mathrm{O}$ aplicativo para gerenciamento, aquisição e armazenamento de imagens foi desenvolvido para sistemas operacionais android, utilizando a linguagem de programação Dart e a ferramenta de desenvolvimento de software Flutter [22]. Possui uma interface minimalista de fácil utilização (Fig. 3).

Inicialmente, o sistema solicita ao usuário a identificação do proprietário e do animal a ser diagnosticado. Na presente versão os dados informativos são inseridos por digitação, mas existe previsão para leitura do brinco de identificação do animal (por reconhecimento de imagem). Em sequência, o aplicativo acessa a câmera fotográfica do dispositivo, permitindo a captura da imagem da região do entorno do olho do animal. Após a aquisição da imagem, é habilitada uma ferramenta de recorte manual (com possibilidade de zoom) para que o usuário selecione a região de interesse. Em versão futura, pretende-se incluir uma etapa de segmentação automática da mucosa conjuntiva. Finalmente, a imagem, recorte e 
informações do animal são armazenadas no dispositivo para posterior processamento. $\mathrm{O}$ aplicativo também possui um sucinto manual digital integrado com explicações de utilização.

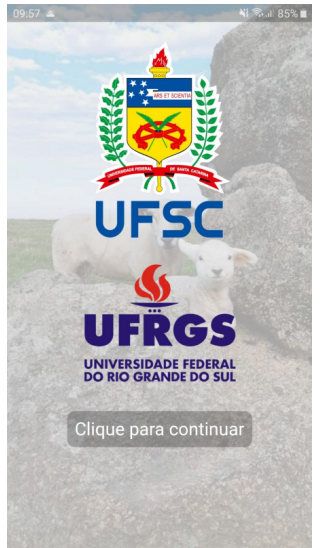

(a)

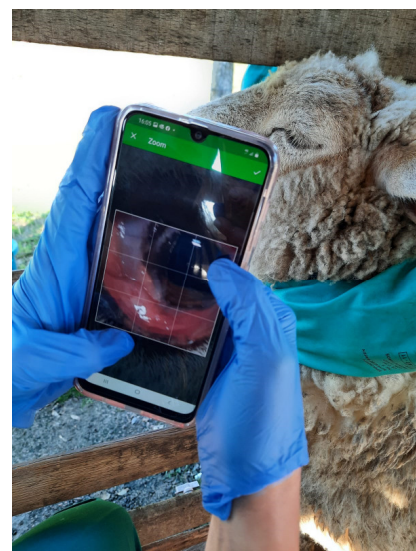

(b)
Fig. 3. Interface do aplicativo. (a) Tela de apresentação; (b) Etapa de recorte.

\section{B. Classificador de Tratamento Famacha ${ }^{\mathcal{O}}$}

O objetivo do classificador desenvolvido é a determinação da classe de tratamento do animal a partir da aplicação automática do método Famacha ${ }^{\odot}$. O recorte da imagem da mucosa conjuntiva ocular do animal em análise, obtido pelo aplicativo descrito na seção III.A, é classificado como: (A) saudável (graus 1 e 2); e (B) anêmico (graus 3, 4 e 5).

O classificador baseia-se em uma rede neural artificial feedforward, do tipo perceptron de múltiplas camadas (multilayer perceptron - MLP) completamente conectada [23]. A versão atual foi desenvolvida para computadores pessoais utilizando a linguagem de programação Python sob o ambiente de desenvolvimento Spyder. Foram utilizadas as bibliotecas TensorFlow, Scikit-Learn e Keras. O otimizador escolhido foi o Adabound [24] (taxa de aprendizado inicial de $10^{-4}$ e final de 0,1) e função de perda Binary Cross-Entropy [25], tendo a acurácia como métrica de avaliação.

Utilizou-se um modelo sequencial de rede neural com duas camadas ocultas. A camada de entrada conta com $N_{1}$ neurônios, enquanto que as duas camadas ocultas utilizam $N_{2}$ neurônios cada. Ambas fazem uso da função de ativação ReLU [26] de maneira a evitar o problema de gradiente enfraquecido. A camada de saída possui 2 neurônios e função de ativação SoftMax.

Posteriormente, tem-se como objetivo realizar a integração do classificador desenvolvido ao aplicativo.

\section{MATERIAIS E MÉTODOS}

Nesta seção são apresentados os materiais e métodos utilizados para a avaliação do classificador descrito na seção III.B.

\section{A. Banco de Imagens}

As imagens utilizadas foram obtidas, sob permissão, durante procedimentos de inspeção de rotina, utilizando o método Famacha ${ }^{\odot}$, em cinco rebanhos:
- Propriedade 1 (Porto Alegre - RS): 23 ovinos, cruzas de raças Texel, Corriedale e Hampshire Down.

- Propriedade 2 (Eldorado do Sul - RS): 38 ovinos cruzas de raças Corriedale, Île-de-France e Hampshire Down.

- Propriedade 3 (Pelotas - RS): 181 ovinos, cruzas de raças Crioula, Texel, Corriedale e Île-de-France.

- Propriedade 4 (Eldorado do Sul - RS): 66 ovinos cruzas de raças Texel e Corriedale.

- Propriedade 5 (Porto Alegre - RS): 9 ovinos, cruzas de raças Texel, Corriedale e Hampshire Down.

Esse estudo foi aprovado pelo Comitê de Ética no Uso de Animais (CEUA) da Universidade Federal do Rio Grande do Sul, projeto número 39150.

Como resultado foram coletadas 317 imagens de mucosa conjuntiva, distribuídas pelos graus Famacha ${ }^{\odot}$ de acordo com a TABELA II.

TABELA II. NÚMERO DE IMAGENS PARA CADA CLASSE E GRAU FAMACHA.

\begin{tabular}{|c|c|c|}
\hline Classe & Grau & Número de imagens \\
\hline \multirow{2}{*}{ A - Saudável } & 1 & 90 \\
\cline { 2 - 3 } & 2 & 123 \\
\hline \multirow{3}{*}{ B - Anêmico } & 3 & 75 \\
\cline { 2 - 3 } & 4 & 17 \\
\cline { 2 - 3 } & 5 & 12 \\
\hline
\end{tabular}

\section{B. Treinamento da Rede Neural}

Inicialmente, os recortes do banco de imagens disponível foram redimensionados para uma matriz de $N \times N$ pixels e, em sequência, vetorizados. Foi utilizado o formato RGB e, posteriormente, as imagens passaram por uma etapa de préprocessamento utilizando a função preprocess_input do Keras.

Os recortes foram separados em um conjunto de treinamento contendo $70 \%$ das imagens de cada grau (1-5) e classe $(\mathrm{A}, \mathrm{B})$ e um conjunto de teste com $30 \%$. Para a classe B (graus 3, 4 e 5) foi utilizada a técnica de data augmentation [27], utilizando-se a função ImageDataGenerator do Keras.

No treinamento da rede neural foram utilizadas 40 épocas e um conjunto de validação constituído de $30 \%$ do conjunto de treinamento. $\mathrm{O}$ peso de cada classe foi balanceado automaticamente utilizando a especificação class_weight. Nenhum processo adicional de extração de características foi utilizado. Foram avaliadas as seguintes combinações de hiperparâmetros: $N \in\{10,30,100\} ; N_{1} \in\{16,32\} ; N_{2} \in\{16$, $32,64\}$; com e sem aumento de dados por geração de amostras sintéticas.

\section{Avaliação de Desempenho}

A avaliação de desempenho foi realizada na forma dos critérios de acurácia, precisão e sensibilidade, respectivamente definidos como:

$$
\text { Acurácia } \equiv \frac{V P+V N}{V P+V N+F P+F N},
$$


TABELA III. RESULTADOS EXPERIMENTAIS.

\begin{tabular}{ccc|cccc}
\hline$N$ & $N_{1}$ & $N_{2}$ & Acurácia $(\%)$ & Precisão $(\%)$ & Sensibilidade $(\%)$ & $\mathrm{F}_{1}(\%)$ \\
\hline 30 & 16 & 16 & 79 & 78 & 81 & 79 \\
30 & 16 & 32 & 78 & 79 & 78 & 78 \\
30 & 16 & 64 & 78 & 79 & 78 & 78 \\
30 & 32 & 16 & 76 & 78 & 75 & 76 \\
30 & 32 & 32 & 79 & 78 & 81 & 79 \\
30 & 32 & 64 & 79 & 80 & 80 & 80 \\
100 & 16 & 16 & 79 & 83 & 75 & 79 \\
100 & 16 & 32 & 76 & 78 & 75 & 76 \\
100 & 16 & 64 & 78 & 78 & 79 & 79 \\
100 & 32 & 16 & 78 & 78 & 79 & 79 \\
100 & 32 & 32 & 81 & 83 & 79 & 81 \\
100 & 32 & 64 & 83 & 85 & 82 & 83 \\
\hline
\end{tabular}

$$
\text { Precisão } \equiv \frac{V P}{V P+F P}
$$

$$
\text { Sensibilidade } \equiv \frac{V P}{V P+F N},
$$

obtidos a partir da matriz de confusão, em que VP, VN, FP e FN correspondem, respectivamente, ao número de verdadeiros positivos, verdadeiros negativos, falsos positivos e falsos negativos [28]. A medida $F_{1}$ é uma combinação das medidas de sensibilidade e precisão:

$$
F_{1} \equiv 2 \frac{\text { Sensibilidade } \times \text { Precisão }}{\text { Sensibilidade }+ \text { Precisão }},
$$

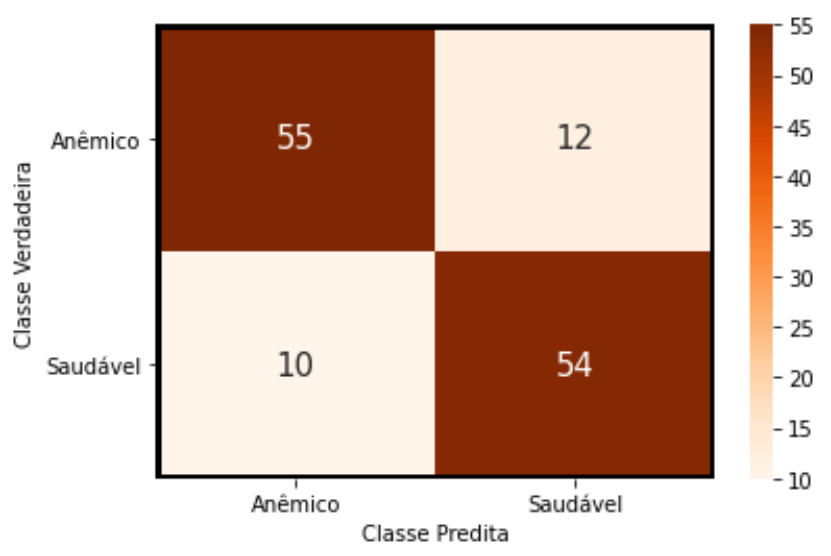

Fig. 4. Matriz de confusão do conjunto de teste, para os parâmetros de melhor desempenho.

\section{RESUltados}

Nesta seção, são apresentados os resultados obtidos na análise de desempenho do classificador proposto.

A TABELA III apresenta um resumo dos resultados obtidos para os diversos conjuntos de parâmetros de treinamento. Todos referem-se a simulações com aumento de dados por geração de amostras sintéticas.

A Fig. 4 apresenta a matriz de confusão obtida pelo classificador para o conjunto de teste associado ao conjunto de parâmetros com maior acurácia (última linha da TABELA III). Os resultados obtidos nessa configuração indicam valores de $83 \%$ de acurácia, $85 \%$ de precisão e $82 \%$ de sensibilidade.

\section{DISCUSSÃO}

Atualmente, o método Famacha ${ }^{\odot}$ é o tratamento mais utilizado no mundo para o controle de infecções parasitárias em pequenos ruminantes.

Uma extensa revisão bibliográfica indica a existência de apenas duas referências que tratam da automatização desse processo, com apresentação de resultados (google scholar, Ieeexplore, Science Direct) [29]. Em [18] foi utilizado um classificador Naive Bayes para duas classes "Não Tratar" (graus 1 e 2) e "Tratar" (graus 3, 4 e 5), resultando em uma acurácia de $66 \%$. Uma limitação importante desse trabalho é a inexistência de animais com grau 5. Em [20] foram utilizados classificadores do tipo máquina de vetor de suporte (support vector machines - SVM) e k-vizinhos mais próximos $(k$ nearest neighbors - KNN) para três classes de saída (saudável, limítrofe e anêmico). O melhor classificador obtido em [20] foi um KNN, apresentando valores de $75 \%$ de acurácia, $75 \%$ de precisão e $77 \%$ de sensibilidade. Entretanto, para uso pelo produtor considera-se desnecessária a determinação da classe limítrofe, visto a recomendação de tratamento.

Em comparação com os resultados previamente apresentados na literatura, os obtidos neste trabalho apresentam um incremento de pelo menos $8 \%$ tanto na acurácia quanto na precisão. Estudos futuros devem verificar a compatibilidade desses resultados com a variabilidade encontrada no diagnóstico provido por diferentes profissionais.

O tempo médio de execução do classificador (por imagem) para a configuração de maior desempenho (última linha da TABELA III) em um computador pessoal com processador Intel@ Core ${ }^{\mathrm{TM}}$ i5-3337U CPU @ 1.80GHz, sem otimização de código, foi de 40 milissegundos.

A disponibilidade de memória e capacidade computacional de dispositivos móveis celulares, assim como o tempo de execução do classificador, são fatores limitantes ao aumento do número de neurônios na rede escolhida. Dessa forma, para que seja obtido um desempenho maior alternativas de pré- 
processamento e extração de características devem ser analisadas. Adicionalmente, a avaliação em campo do sistema de aquisição e recorte de imagens indica a pertinência da inclusão de um subsistema de segmentação automática da mucosa do animal.

Ao final do processo de integração dos subsistemas apresentados neste trabalho, espera-se que o sistema proposto permita a aplicação confiável da técnica Famacha ${ }^{\odot}$ pelos próprios produtores rurais ou seus funcionários, sem necessidade de treinamento prévio ou deslocamento de um técnico especializado. Dessa forma, o aplicativo desenvolvido possibilitará a redução dos custos de manejo de pequenos ruminantes em pequenas propriedades rurais, especialmente nas de difícil acesso, visto que para um controle parasitário efetivo é necessário que o procedimento seja empregado regularmente, de forma mensal ou quinzenal, dependendo da época do ano.

\section{CONCLUSÕES}

Este trabalho apresentou uma proposta de sistema para aquisição de imagens da mucosa conjuntiva e classificação da indicação de tratamento em relação ao grau de infestação parasitária em pequenos ruminantes. Uma estratégia baseada em redes neurais artificiais foi utilizada, resultando em uma acurácia de $83 \%$, na determinação de duas classes (saudável e anêmico), segundo o método Famacha ${ }^{\odot}$. Os resultados obtidos indicam a possibilidade de automatização do processo de classificação com a diminuição dos custos envolvidos ao produtor de pequenos ruminantes.

\section{AGRADECIMENTOS}

À Raquel Fraga e Silva Raimondo pelo apoio na coleta de dados. Ao programa institucional de iniciação científica do $\mathrm{CNPq}$ da Universidade Federal de Santa Catarina pelo suporte financeiro. Este trabalho foi parcialmente financiado pelo CNPq (315020/2018-0)

\section{REFERÊNCIAS}

[1] R V. M. M. Costa, S. V. D. Simões, F. Riet-Correa, "Doenças parasitárias em ruminantes no semiárido brasileiro", Pesquisa Veterinária Brasileira, v. 29, pp. 563-568, 2009.

[2] J. F. J. Torres-Acosta, P. Mendoza-De-Gives, A. J. Aguilar-Caballero, J. A. Cuéllar-Ordaz, "Anthelmintic resistance in sheep farms: update of the situation in the American continent", Veterinary Parasitology, v. 189, pp. 89-96, 2012.

[3] F. Riet-Correa, A. L. Schild, M. D. C. Mendez, R. A. A. Lemos, Doenças de ruminantes e equinos, $2^{\mathrm{a}}$ ed., 2007, p. 90.

[4] M. B. Molento, C. J. Veríssimo, A. T. Amarante, J. A. Wyk,. A. C. S. Chagas, J. V. Araújo, F. A. Borgeset "Alternativas para o controle de nematoides gastrintestinais de pequenos ruminantes", Arquivos do Instituto Biológico, v. 80, n. 2, p.253-263, 2013.

[5] J. F. J. Torres-Acosta, H. Hoste, "Alternative or improved methods to limit gastrointestinal parasitism in grazing sheep and goats", Small Ruminant Research, v. 77, pp. 159-173, 2008.

[6] S. Preston, D. Piedrafita, M. Sanderman, S. Cotton, "The current status of anthelmintic resistance in a temperate region of Australia; implications for small ruminant farm management", Veterinary Parasitology: Regional Studies and Reports, vol. 17, pp. 1-6, 2019.

[7] J. A. Van Wyk, M. O. Stenson, J. S. Van der Merwe, R. J. Vorster, P. G. Viljoen, "Anthelmintic resistance in South Africa: surveys indicate an extremely serious situation in sheep and goat farming", Journal of Veterinary Research, v. 66, pp. 273-285, 1999.
[8] W. E. Pomroy, "Anthelmintic resistance in New Zealand: a perspective on recent findings and options for the future", New Zealand Veterinary Journal, v. 54, pp. 265-270, 2006.

[9] A. C. F. L. Melo, I. F. Reis, C. M. L. Bevilaqua, L. S. Vieira, F. A. M. Echevarria, L. M. Melo, "Nematódeos resistentes a anti-helmínticos em rebanhos de ovinos e caprinos do Estado do Ceará", Ciência Rural, v. 33, pp. 339-344, 2003.

[10] B. Riet-Correa, S. V. D. Simões, F. Riet-Correa, "Sistemas produtivos de caprinocultura leiteira no semiárido nordestino: controle integrado das parasitoses gastrointestinais visando contornar a resistência antihelmíntica", Pesquisa Veterinária Brasileira, v. 33, pp. 901-908, 2013.

[11] P. A. Oliveira, B. Riet-Correa, P. Estima-Silva, A. C. B. Coelho, B. L. Santos, M. A. P. Costa, J. L. Ruas, A. L. Schild, "Multiple anthelmintic resistance in Southern Brazil sheep flocks", Revista Brasileira de Parasitologia Veterinária, v. 26, pp. 901-908, 2017.

[12] M. B. Molento, "Resistência de helmintos em ovinos e caprinos", Congresso Brasileiro de Parasitologia Veterinária, 2004, pp 1-13.

[13] L. Rinaldi, G. Cringoli, "Parasitological and pathophysiological methods for selective application of anthelmintic treatments in goats", Small Ruminant Research, v. 103, pp. 18-22, 2012.

[14] A. Zajac, "How and why to do Famacha ${ }^{\odot}$ scoring", American Consortium for Small Ruminant Parasite Control, Virginia Tech., 2014.

[15] A. P. Minho, M. M. B. Molento, "Método FAMACHA: Uma técnica para prevenir o aparecimento da resistência parasitária", Circular Técnica da Embrapa, n. 46, pp. 1-6, 2014.

[16] A. C. S. Chagas, M. C. S. Oliveira, C. O. Carvalho, M. B. Molento, "Método FAMACHA: Um recurso para o controle da verminose em ovinos", Circular Técnica Embrapa Pecuária Sudeste, pp. 1-8, 2012.

[17] M. A. M. Fernandes, S. Gilaverte, A. Buzatti, L. K. Sprenger, C. J. A. Silva, M. T. P. Peres, M. B. Molento, A. Monteiro, "Método FAMACHA para detectar anemia clínica causada por Haemonchus Contortus em cordeiros lactentes e ovelhas em lactação", Pesquisa Veterinária Brasileira, v. 35, n. 6, pp. 525-530, 2015.

[18] G. Demoliner, R. J. Fernandes Alves, "Anemimetro: app móvel para implementação do método Famacha", Unoesc \& Ciência, v. 8, n. 1, pp. 25-32, 2017.

[19] T. J. Brinker et al., "A convolutional neural network trained with dermoscopic images performed on par with 145 dermatologists in a clinical melanoma image classification task", European Journal of Cancer, v. 11, pp 148-154, 2019.

[20] W. Ribeiro, Uma Metodologia para Detectar Anemia em Pequenos Ruminantes, Monografia de Conclusão de Curso, Curso de Ciências da Computação, Universidade Federal do Maranhão, 2018.

[21] Y. R. Sivathanu, J. Lim, Method and a mobile app for use with a mobile device to accurately quantify blood hemoglobin in mammals, US Patent 2016/0089062 A1, 2016.

[22] R. Payne, Beginning App Development with Flutter, Apress, 2019.

[23] S. Haykin, Neural Networks and Learning Machines, terceira edição, Pearson, 2008.

[24] L. Luo, Y. Xiong, Y. Liu, X. Sun, "Adaptive gradiente methods with dynamic bound of learning rate", International Conference on Learning Representations, 2019, pp 1-21.

[25] D. Godoy, "Understanding binary cross-entropy/log loss: A visual explanation", Towards Datascience, 2018.

[26] V. Nair, G. E. Hinton, "Rectified linear units improve restricted Boltzmann machines", International Conference on Machine Learning, 2010, pp. 807-814.

[27] C. Shorten, T. M. Khoshgoftaar, "A survey on image data augmentation for deep learning", Journal of Big Data, v. 6, pp. 1-48, 2019.

[28] K. Faceli, A. C. Lorena, J. Gama, T. A. Almeida, A. C. P. L. F Carva, Inteligência Artificial: uma abordagem por aprendizado de máquina, LTC, 2011.

[29] R. García, J. Aguilar, M. Toro, A. Pinto, P. Rodríguez, “A systematic literature review on the use of machine learning in precision livestock farming", Computers and Electronics in Agriculture, v. 179, pp. 1-12, 2020 . 